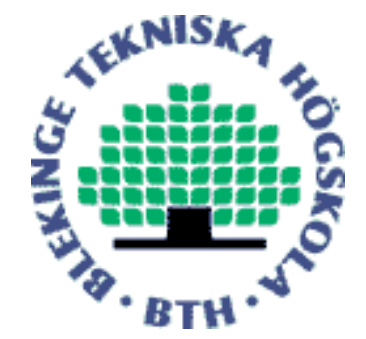

Copyright (C) 2014 IEEE.

Citation for the published paper:

On Bistatic Forward-looking SAR Imaging

Viet Thuy Vu, Mats Pettersson

Geoscience and Remote Sensing Symposium (IGARSS)

2014 Quebec

This material is posted here with permission of the IEEE. Such permission of the IEEE does not in any way imply IEEE endorsement of any of BTH's products or services Internal or personal use of this material is permitted. However, permission to reprint/republish this material for advertising or promotional purposes or for creating new collective works for resale or redistribution must be obtained from the IEEE by sending a blank email message to pubs-permissions@ieee.org.

By choosing to view this document, you agree to all provisions of the copyright laws protecting it. 


\title{
ON BISTATIC FORWARD-LOOKING SAR IMAGING
}

\author{
Viet T. Vu and Mats I. Pettersson* \\ Blekinge Institute of Technology, Campus Gräsvik, 37179 Karlskrona, Sweden
}

\begin{abstract}
Left/right ambiguity and low angular (azimuth) resolution are severe problems for monostatic forward-looking SAR imaging. It is strongly believed that these technical issues can definitely be solved with bistatic forward-looking SAR. The analysis presented in this paper points out that the left/right ambiguity problem still exits. However, an appropriate selection of the position of bistatic base line and antenna beamwidth allows us to conceal it. The paper also gives some recommendations which can be considred for the forward-looking SAR imaging.
\end{abstract}

Index Terms - forward-looking, SAR, backprojection, left/right ambiguity

\section{INTRODUCTION}

One of the crucial applications of forward-looking SAR imaging can be found in aircrafts' landing systems [1]. In severse weather like dense fog, the visual ability of pilots is significantly limited and a forward-looking SAR imaging system will therefore be helpful for a pilot to land the aircraft securely. For monostatic SAR, forward-looking imaging is challenged by left/right ambiguity and low angular (azimuth) resolution. The former is due to the fact that the Doppler histories of the scatterers in an illuminated SAR scene locating symmetrically about the flight track are identical while the latter is resulted by the small gradient of the Doppler frequency in the flight direction. Several approaches for such problems have been introduced in 1990's for monostatic SAR. In an early approach, the Doppler beam sharpening (DBS) mode is considered for the forward-looking SAR system [2]. It is necessary for the antenna of the system to be with specific aperture illumination functions in order to maximize angular resolution. A forward-looking SAR mode with two receiving antennas and one transmitting antenna on a radar platform is introduced in [3] and it is declared that the left/right ambiguity problem of forward-looking SAR imaging can be solved in theory.

The emergence of bistatic SAR opens new opportunities for forward-looking SAR imaging. It is strongly believed that

\footnotetext{
${ }^{*}$ The authors would like to thank the KK-Foundation for the financial support in this research project, the Swedish Defence Research Agency, Saab Bofors Dynamics, Saab Electronic Defence Systems and RUAG Space for their cooperation.
}

the technical issues of forward-looking SAR imaging can definitely be solved with bistatic forward-looking SAR. A large number of research on bistatic forward-looking SAR imaging has been announced recently including series of experiments and processing techniques. One of the first forward-looking SAR imaging experiments was carried by FGAN in 2004 [4]. In 2009, an experiment was executed with TerraSAR$\mathrm{X}$ and PAMIR in order to study the feasibility of forward or backward direction [5] and recently another one using a receiver on board an ultra light aircraft and a transmitter on a moving truck to illuminate the runway was implemented [6]. The commonly used frequency-domain processing techniques extended for bistatic cases such as Range Doppler, Range Migration and Chirp Scaling have also been used for the forward-looking SAR imaging application. Among them, only Range Doppler is shown to work with the measured forward-looking SAR data [4]. However, the lack of flexibility of the frequency-domain algorithms may prevent us from investigating into bistatic forward-looking SAR imaging in detail.

The bistatic time-domain algorithms developed recently do not suffer from such inflexibility [7]. As stated in [7], these algorithms inherit the time-domain characteristics such as unlimited scene size, real-time processing, local processing, manageable motion compensation, and large RM handling. For bistatic cases, they are proved to be not limited by any bistatic geometry. In other words, the operation of these algorithms are independent of the speeds, the flight tracks, and the flight altitudes of transmitter and receiver platforms. They are therefore definitely available for bistatic forwardlooking SAR imaging.

The purpose of the paper is to use the new developed bistatic time-domain algorithms to investigate into the problems of bistatic forward-looking SAR imaging in detail.

\section{SAR ALGORITHMS AND AVAILABLITY FOR BISTATIC FORWARD-LOOKING}

Backprojection is interpreted as a linear and direct transformation process from radar echoes into a complex SAR image. If the range-compressed radar signal is given by $g(t, \tau)$ where $t$ and $\tau$ indicate azimuth-time (slow-time) and range time (fast-time), respectively, the superposition of backprojected radar echoes to reconstruct a sample $\left(x_{m}, y_{n}\right)$ of the 


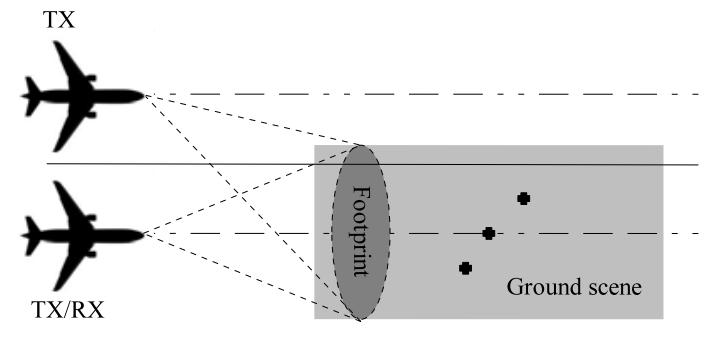

Fig. 1. Bistatic geometries - quasi-monostatic and azimuth invariant.

SAR image in the ground plane $(x, y)$ is mathematically represented by the following integral [7]

$$
h\left(x_{m}, y_{n}\right)=\int_{-\frac{t_{i}}{2}}^{+\frac{t_{i}}{2}} g\left(t, \frac{R_{m, n}(t)}{\mathrm{c}}\right) d t
$$

where $t_{i}$ is the integration time, $\mathrm{c}$ is the speed of light and $R_{m, n}(t)$ is bistatic range. The calculation of $R_{m, n}(t)$ is applied for all aperture positions and all SAR image elements and thus requires a high computational burden.

The fast time-domain bistatic algorithms do not transform radar echoes into a SAR image but another intermediate form and then interpolate it to a SAR image. This allows reduce processing time significantly. Beam formed in the first stage of the fast time-domain bistatic algorithms introduced in [7] is such an intermediate form.

To prove their availability for bistatic forward-looking SAR imaging and then investigate into the problems of bistatic forward-looking SAR imaging, two bistatic geometries, quasi-monostatic and azimuth-invariant, are here considered. Please note that this consideration is only for convenience. These geometries are illustrated in Fig. 1.

The bistatic forward-looking SAR system is simulated with the parameters summarized in Table 1 . The parameters are similar to the ones of LORA-GMTI except the coordinates of the platform(s).

The SAR scene is simulated by a long runway that three targets are present. Their radar cross sections are normalized to 1 . The arrangement of these targets is shown in Fig. 1 and this arrangement is supposed to be reasonable for investigating into the problems of bistatic forward-looking SAR imaging later.

\subsection{Quasi-monostatic}

For the quasi-monostatic case, transmitter and receiver are mounted on a single platform and separated by a distance of $3 / 2$. This bistatic configuration has been used in LORA for ground moving target indication (GMTI) purposes. The antennas are assumed to be narrowbeam and the antenna
Table 1. Parameters of simulations

\begin{tabular}{|c|c|c|}
\hline Parameter & TX/RX & TX \\
\hline Frequency range & $307-333 \mathrm{MHz}$ & $307-333 \mathrm{MHz}$ \\
\hline Platform speed & $130 \mathrm{~m} / \mathrm{s}$ & $130 \mathrm{~m} / \mathrm{s}$ \\
\hline Flight altitude & $600 \mathrm{~m}$ & $600 \mathrm{~m}$ \\
\hline PRF & $1300 \mathrm{~Hz}$ & $1300 \mathrm{~Hz}$ \\
\hline Aperture step/length & $0.1 / 600 \mathrm{~m}$ & $0.1 / 600 \mathrm{~m}$ \\
\hline Integration time $t_{i}$ & $5 \mathrm{~s}$ & $5 \mathrm{~s}$ \\
\hline Minimum range $r_{0}$ & $1000 \mathrm{~m}$ & $1071 \mathrm{~m}$ \\
\hline Antenna separation $d$ & $1.4 \mathrm{~m}$ & $384 \mathrm{~m}$ \\
\hline
\end{tabular}

beams only illuminate the runway. The parameters given in the second column of Table 1 are used to simulate the quasimonostatic SAR system. In this case, the traveling distance of a radar pulse can be approximated by the two-way traveling distance of that radar pulse with respect to the center of the transmitter and receiver. The SAR processing can even be relied on the monostatic SAR algorithms. However, we still use the time-domain bistatic SAR algorithm to form the SAR image in the ground plane.

Fig. 2(a) shows the SAR image of the ground scene (256 $\mathrm{m} \times 1024 \mathrm{~m})$. As observed, the SAR image shows 6 targets instead of 3 as simulated and they are symmetric with respect to the center of the SAR image. This is caused by the left/right ambiguity problem in the forward-looking mode. The SAR image also show the poor resolution in azimuth which is also the inherent problem of SAR systems working in this mode. Due to the poor resolution, two targets in the middle are merged together and we cannot distinguish them by eyes.

\subsection{Azimuth-variant}

For the azimuth-variant case, transmitter and receiver are carried by two separate platforms. Their flight tracks are parallel and the platforms' speeds are identical. This bistatic configuration has been used in the LORAMbis UHF/VHF experiments for FOPEN. The narrowbeam antennas are also assumed here and the antenna beams only illuminate the runway. The transmitter is simulated with the parameters given in the third column of Table 1 whereas the motion parameters given in the second column are used for the simulation of the receiver. We use the same time-domain bistatic SAR algorithm to reconstruct the ground scene.

Fig. 2(b) shows the SAR image of the same ground scene $(256 \mathrm{~m} \times 1024 \mathrm{~m})$. The SAR image shows exactly 3 targets. These targets are well focused and locate at the correct coordinates as simulated. The left/right ambiguity problem in the forward-looking models not detected in the SAR image. 

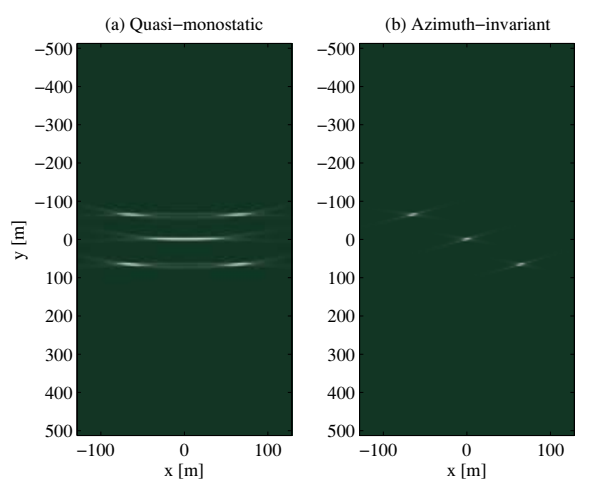

Fig. 2. Forward-looking bistatic SAR images of runway.

The SAR image also show that the resolution in azimuth is improved significantly in comparison to the azimuth resolution obtained with the quasi-monostatic geometry. We can also recognize this if we compare the middle part of the SAR images given in Fig. 2(a) and (b).

An explanation about the improvement in azimuth resolution can be found, e.g. in [8], and this will not be further considered in this study. Hence, the left/right ambiguity problem in bistatic forward-looking SAR imaging will be considered in the next section.

\section{LEFT/RIGHT AMBIGUITY PROBLEM IN BISTATIC FORWARD-LOOKING}

As mentioned in the previous section, the left/right ambiguity problem in the forward-looking mode has not been detected in the SAR image given in Fig. 2(b) as the targets are well focused and locate in the correct coordinates with the azimuthinvariant geometry. If we assume that the ground scene is now not only the runway but also the area in the left hand side, i.e. the same side with the transmitter platform. We also assume that there are three targets on the runway and there is one more new target locating somewhere in the left hand side area. The normalized radar cross section of the new target is 2 (larger than three others). The ground scene is illuminated by the same bistatic SAR system as Section II-B. However, the narrowbeam antennas are now replaced by widebeam antennas. This allows the bistatic SAR system illuminate both the runway and the left hand side area. The same time-domain bistatic SAR algorithm is applied for SAR image formation. However, the size of SAR image is increased to $640 \mathrm{~m} \times 1024$ $\mathrm{m}$ instead of $256 \mathrm{~m} \times 1024 \mathrm{~m}$ since a larger SAR scene is here considered.

Fig. 3 shows the SAR image of the same ground scene $(640 \mathrm{~m} \times 1024 \mathrm{~m})$ which includes the runway with three weak reflected targets given in Fig. 2(b) and its left hand side area with one strong reflected target. Similar to the SAR image given in Fig. 2(b), the resolution in azimuth is improved significantly in comparison to the azimuth resolution obtained

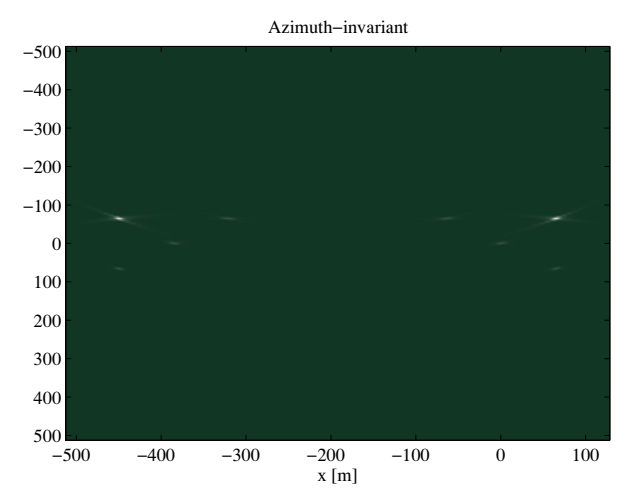

Fig. 3. Azimuth-variant bistatic SAR image of a larger scene.

with the quasi-monostatic geometry. The SAR image shows 8 targets which are well focused. Three of four targets with weak reflection in the right hand side of the SAR image locate at the correct coordinates as simulated. The fourth one which is not included in the simulation of the right hand side SAR scene. There are another four targets in the left hand side area of the runway in which only strong reflected one is included in the left hand side SAR scene simulation. The targets in the left and right hand sides are symmetric with respect to the line $x=-192 \mathrm{~m}$. Based on Fig. 2(b), we can predict that three weak reflected targets with correct coordinates in the right hand side of the SAR image and the strong reflected one in the top left one are the simulated targets. The others are only the images of the simulated targets. This phenomenon shows that the left/right ambiguity problem is still available in the forward-looking mode regardless of the azimuth-invariant geometry. The line $x=-192 \mathrm{~m}$ is identical with the set of central points between the transmitter and the receiver platforms (two platforms are separated by $d=384 \mathrm{~m}$ ) and in this paper we call the bistatic base line.

Hence, bistatic geometries do not ensure the Doppler histories of the scatterers in a SAR scene different or in other words the left/right ambiguity problem can happen with bistatic geometries. As illustrated in Fig. 4 for the azimuthinvariant geometry, the target in the left hand side of the SAR scene (black cross) and the one in the right hand side (dark gray cross) place symmetrically about the bistatic base line and thus have exactly the same Doppler histories when the platforms move parallel with the same speed. For a general geometry, we can also find targets which have approximately the same Doppler histories. The effects of the left/right ambiguity problem to a SAR scene of interest can be significant. To illustrate this statement, we simulate a bistatic SAR system with the general geometry. We use the same parameters given in Table 1 except that the speed of the transmitter platform increases $20 \%$, i.e. $156 \mathrm{~m} / \mathrm{s}$, and the direction of movement is $15^{\circ}$ instead of $0^{\circ}$ as illustrated by the gray line in Fig. 4 .

The SAR image of the same ground scene including the runway with three weak reflected targets and its left hand side 


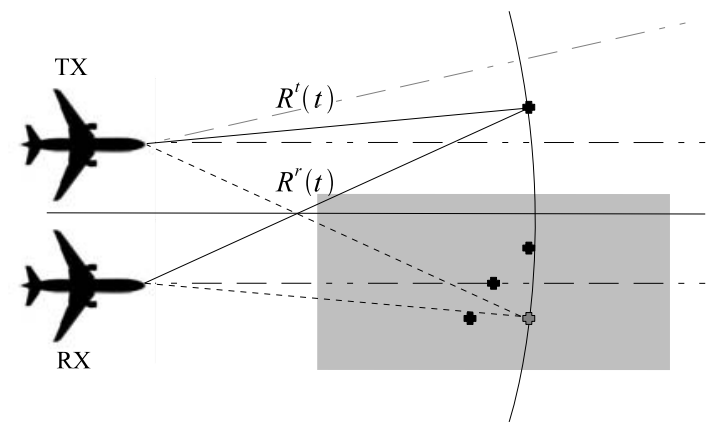

Fig. 4. Left/right ambiguity problem in bistatic geometry.

area with one strong reflected target is shown in Fig. 5. The true targets (three in the right hand side and one in the left hand side) are well focused in the SAR image at the correct coordinates as simulated. The images of the three targets in the right hand side lie in the other side of the bistatic base line and are migrated from the SAR image due to the modification of the transmitter platform's motion parameters. The image of the other appears in the right hand side of the SAR image due to the left/right ambiguity problem. However, it is not well focused as the true target in the left hand side due to the mismatch of the Doppler histories of the true target and its image. Due to the strong reflection, the intensity of the image of the target is still stronger than the ones of the true targets in the right hand side.

\section{RECOMMENDATIONS}

The investigations in the previous section show that the left/right ambiguity problem in bistatic forward-looking SAR imaging depends on position of bistatic base line and antenna beamwidth. This is valid for all bistatic geometries. The recommendations for antenna and geometry are given as follows:

- The bistatic base line of a bistatic forward-looking SAR system must be beyond the area of interest. We can say the border of an area of interest is the limit for the bistatic base line.

- The beamwidths of transmitting receiving antennas should be narrow enough so that their footprints should be inside the bistatic base line.

The violation one among two conditions leads to the left/right ambiguity problem. As a result of the left/right ambiguity problem, we will not get a correct SAR image of an area of interest or a SAR image of unwanted area may obscure a SAR image of the area of interest.

The above recommendations lead to two possibilities to conceal the left/right ambiguity problem in bistatic forwardlooking SAR. Hence, we can largely separate transmitter and receiver or we can use small beamwidth antennas. However, a large separation between transmitter and receiver is highly

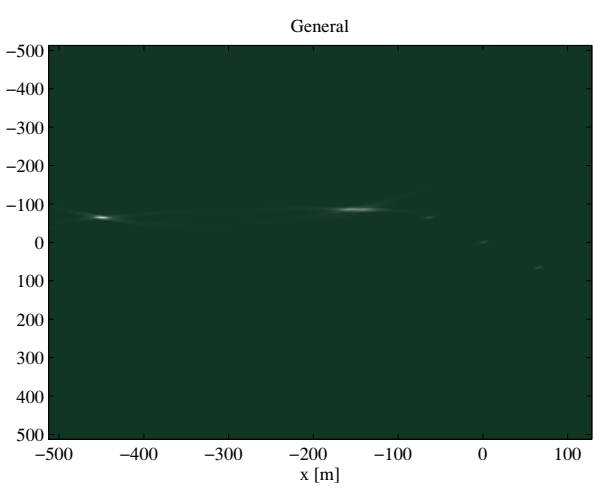

Fig. 5. General bistatic SAR image of a larger scene.

recommended to simplify the selection of the antennas as well as the spatial synchronization, i.e. the overlap of two antenna footprints in bistatic geometries.

\section{REFERENCES}

[1] L.Q. Bui, et. al., "Test results of an experimental autonomous aircraft landing system utilizing a $94 \mathrm{GHz} F \mathrm{FM}$ $\mathrm{CW}$ imaging radar", in IEEE MTT-S International Microwave Symposium, Atlanta, GA, June 1993, pp. 857860.

[2] A. K. Löhner, "Improved azimuthal resolution of forward looking SAR by sophisticated antenna illumination function design", Proc. IEE Radar Sonar Navig., vol. 145, no. 2, pp. 128-134, 1998.

[3] S. Dai and W. Wiesbek, "High resolution imaging for forward looking SAR with multiple receiving antennas", in Proc. IEEE IGARSS, Honolulu, HI, July 2000, pp. 22542256.

[4] J. Balke, "Field test of bistatic forward-looking synthetic aperture radar," in Proc. IEEE RadarCon, Washington, DC, May 2005, pp. 424-429.

[5] I. Walterscheid, et. al., "Potential and limitations of forward-looking bistatic SAR", in Proc. IEEE IGARSS, Honolulu, HI, July 2010, pp. 216-219.

[6] I. Walterscheid and B. Papke, "Bistatic forward-looking SAR imaging of a runway using a compact receiver on board an ultralight aircraft", in Proc. IEEE IRS, Dresden, Germany, June 2013, pp. 461-466.

[7] V. T. Vu, et. al., "Fast time-domain algorithms for UWB bistatic SAR processing", IEEE Trans. Aerosp. Electron. Syst., vol. 49, no. 3, pp. 1982-1994, 2013.

[8] I. Walterscheid, et. al., "Potential and limitations of forward-looking bistatic SAR", in Proc. IEEE IGARSS, Honolulu, HI, July 2010, pp. 216-219. 